\title{
Isolation and Identification of Gram-Negative Bacteria from Oral Cancer Site Infections and Study of Their Antibiotic Resistance Pattern
}

\author{
Samiha Ashreen ${ }^{1}$, Akash Ahmed², Nadimul Hasan³, Waheed Akhtar ${ }^{4}$, M. Mahboob Hossain²* \\ ${ }^{1}$ Biotechnology Program, Department of Mathematics \& Natural Sciences, Brac University, 66 Mohakhali, Dhaka 1212, Bangladesh, ${ }^{2}$ Microbiology Program, \\ Department of Mathematics \& Natural Sciences, Brac University, 66 Mohakhali, Dhaka 1212, Bangladesh, ${ }^{3}$ Deptartment of Dental \& Faciomaxillary Surgical \\ Oncology, National institute of Cancer Research \& Hospital, Dhaka, Bangladesh, ${ }^{4}$ Department of Radiation Oncology, National institute of Cancer Research \& \\ Hospital, Dhaka, Bangladesh
}

\begin{abstract}
Cancer is one of the non-communicable lethal diseases which affects millions of people worldwide and is responsible for the majority of global deaths. Among the types of cancers occurring in humans, oral cancer has a higher incident rate which is significantly alarming. Oral cancer sites are associated with increased risk of metastasis, along with making the cancer site susceptible to infection by a vast number of opportunistic pathogens. Gram-negative opportunistic bacilli can dwell in these cancer ulcer sites since the patient becomes immune compressed due to radiotherapy and chemotherapy treatments, which prolong the infection. The present study was undertaken to find the most prevalent gram-negative bacilli in oral cavity cancer sites. Swab samples from 50 oral cancer patients were taken to check the presence of the opportunistic organisms. While the control group was set with people with no cancer, only 4 bacteria were isolated from 40 healthy volunteers. Among the isolates from both test group and control group, the most prevalent organism was Klebsiella species 37(45\%), followed by Pseudomonas species 29 $(34.5 \%)$ and Proteus species 8(9.5\%). The least prevalent was Escherichia coli 5(6\%). The isolates were all taken for antibiotic sensitivity testing (AST) against 15 antibiotics from 11 different groups used in hospitals. Results of the AST were as follows: $100 \%$ of the isolates from cancer patients were resistant to vancomycin, clindamycin, amoxicillin, penicillin G, and metronidazole. It was followed by azithromycin, where $86.25 \%$ of the isolates were resistant to it. The least resistance was seen against aminoglycoside antibiotic group, amikacin $(6.25 \%)$, then gentamicin having a resistance percentage of 7.5. A substantial amount of qualitative data on medical history, clinical examination, and treatment, etc. was documented and presented in this study.
\end{abstract}

Keywords: Oral cancer, Gram-negative bacteria, Opportunistic infection, Immunocompromised, Antibiotic resistance

\section{Introduction}

Cancer is currently a major global public health issue. Development of cancer cell and survival is a multifactor process that involves genetic mutation of normal cells along with physiological changes of normal body cells and the body's defense mechanism. In Asian countries naming India, Indonesia, Philippines, Vietnam, and China more than 3 million people are expected to be diagnosed with cancer each year ${ }^{1}$. Oral cancer, globally, is the sixth most common cancer $^{2}$ and is a major problem in regions where tobacco habits, in the form of chewing and/or smoking, with or without alcohol intake, are common. Cancer can take place in the anterior tongue, gingival, buccal mucosa, retromolar trigone, hard palate, salivary glands, and even tonsil glands ${ }^{3}$.

Oral cancer arises through a series of histopathologic stages from benign hyperplasia to dysplasia to carcinoma in situ followed by invasive squamous cell carcinoma ${ }^{4}$.The malignancy of oral cancer is preceded by premalignant lesions that are hyperplastic growth. A multistep process involving the accumulation of genetic and epigenetic alterations in key regulatory genes ${ }^{5}$, the hyperplasia can turn into metaplasia and anaplasia. Simultaneous exposure to a wide spectrum of biological agents, chemical substances and physical forces may act as predisposing factors of cancer. External factors of oral cancer include the immoderate consumption of cigarettes, alcohol, tobacco leaf, betel leaf, catechu, etc. Moreover, infection with Herpes virus, Human Papillomavirus, Candida albicans, Treponema pallidum, and even poor oral hygiene can increase the risk of developing oral cancer ${ }^{6}$.

A study revealed that the number of new cancer cases in Bangladesh per year is about 200,000 of which $20 \%$ consists of oral cancer patients ${ }^{7}$. Each year more than 7,000 people in Bangladesh are diagnosed with oral cancer, and $6.6 \%$ of these patients face mortality ${ }^{8}$. An estimation of the International Agency for Research on Cancer (IARC) has reported cancer-related death rate in Bangladesh was $7.5 \%$ in 2005 and it has a chance to increase to $13 \%$ in 2030 . The etiological factors implicated in oral cancer can be used to trace down the relatively higher rate of this disease in this country. People livinginSouth-East Asia, 
the Indian subcontinent, including Bangladesh have a habit of chewing of betel quid with catechu which is dangerous since a carcinogenic compound named arecoline is found in catechu. The quid is usually kept in the mouth for a long period which contributes to increased risk of developing oral cavity cancer. Another prevalent habit of regular smoking and alcohol consumption increases the risk of oral cancer. The most important carcinogens in tobacco smoke are responsible for critical mutations involved in DNA replication which are suspected to play a key role in tobacco-induced head and neck cancer ${ }^{9}$. Moreover, poor oral hygiene along with poor dental status has been suggested to promote neoplasm in the presence of other risk factors.

In the oral cavity, the role of microbes has been linked with malignancies. Microbial population in mouth mucosa differs between healthy and malignant sites which refer to alteration of mouth microbiota due to oral cancer. Studies have identified some microbes such as Treponema pallidum, Human papillomavirus, and Candida albicans as risk factors of oral cancer. Infection in the oral cavity occurs when the balance of bacteria in the body is disrupted, specifically in immunocompromised situations, drugresistant bacteria proliferate over normal microbial flora. The study identified Pseudomonas spp., Klebsiella spp., Escherichia coli, and Proteus spp. as some of the opportunistic gram-negative pathogens that can infect immunocompromised patients. Moreover, the principal treatments for oral cancer being surgical excision, radiotherapy, and chemotherapy ${ }^{10}$ facilitate colonization of opportunistic pathogens in the oral cavity. Additionally, nosocomial infections are commonly found in oral cancer patients after surgical removal of the tumor due to wound exposure during and after the operation.

\section{Materials and Methods}

Study subjects, place and duration: The study involved the collection of both data through a questionnaire and a swab sample from the oral cavity. Data and clinical samples were collected from 50 oral cancer patients taking treatment from the National Institute of Cancer Research and Hospital, Dhaka, Bangladesh, from June 2019 to September 2019. The clinical samples were checked for the presence of infection on their cancer site. Another control group of 40 people was set with healthy volunteers aged over 22 who do not exhibit any signs of cancer.

Collection of the samples: With a sterile autoclaved cotton swab, the pus from the infection site in the oral cavity of oral cancer patients was taken and immediately transferred to sterile test tubes containing autoclaved $0.9 \%$ saline solution. While for the control group with no oral cancer symptoms, the sterile cotton swab was rubbed inside the cheeks, gum area and under the tongue. The tubes were then taken into the laboratory and further experiments were carried out.

Identification of the clinical samples: Taking $501 / 41$ of each sample from the dilution $10^{-2}$ was inoculated into different selective agar media (cetrimide agar and eosin methylene blue agar) performing spread plate technique and inoculated media plates were incubated at $37^{\circ} \mathrm{C}$ for 24 hours. The colony characteristics of the isolates (colony morphology along with their gram reaction) on different selective media were presumptively examined according to the standard microbiological laboratory manual ${ }^{11}$. Later, single colonies were taken and streaked on nutrient agar media for identifying the microorganisms by performing standard biochemical tests (IMVIC test, triple sugar iron test, citrate utilization test, MIU test, catalase test, and oxidase test)

\section{Antibiotic Susceptibility test}

According to the guidelines provided by the Clinical Laboratory Standard Institute (CLSI), the antibiotic susceptibility pattern of the isolates was examined using disc diffusion method ${ }^{12-13}$. The 18 hours fresh culture of the isolates (Pseudomonas spp., Klebsiella spp., Escherichia coli and Proteus spp.) were adjusted to the turbidity of $1 \mathrm{McF}$ arland standards and bacterial suspensions were spread over Mueller-Hinton agar (MHA). The antibiotic disc used in the study included amikacin, gentamicin, imipenem, ceftriaxone, vancomycin, clindamycin, azithromycin, amoxicillin, penicillin- G, amoxiclav, ciprofloxacin, nalidixic acid, tetracycline and metronidazole (oxoid). All the plates were incubated at $37^{\circ} \mathrm{C}$ for $18-20$ hours and the zone of inhibitions was measured (mm).

\section{Results}

Results from the growth on selective media: Of the 50oral cancer patients that participated in the study, 42 patients had infection in their cancer site. Among these 42 patients, 11 patients were post-operative patients and the remaining 31 were pre-operative patients. All the specimens from both pre-operative and postoperative patients exhibited bacterial growth on at least one of the selective media. Of the 40 samples collected from the healthy people were considered as control, only 4 of them showed growth on the selective media used for the isolation of selected gramnegative opportunistic pathogens (Table 1).

Identification of isolates from biochemical tests results: The individual distinct colonies that were found from the selective media were streaked on nutrient agar to observe visual similarities in terms of colony morphology. Of the 84 selected bacterial colonies, 78 were from cancer patients with infection and 2 belonged to cancer patients with no visible infections. Only 4 isolates were retrieved from 40 samples of the control group. Later, biochemical tests were done for further deduction of the microorganism's identity (Table 2).

Antibiotic susceptibility pattern of the isolates: The antibiotics used in the susceptibility testing were selected on the criteria of being regularly used in the hospital for controlling the infection and prophylaxis purpose. The study tried to unveil the efficacy of the 15 commonly available antibiotics from 11 different groups. 
All the isolates from cancer patients were resistant to vancomycin, clindamycin, amoxicillin, penicillin $\mathrm{G}$, and metronidazole. It was followed by azithromycin, where $86.25 \%$ of the isolates were resistant to it. The least resistance was seen against amikacin $(6.25 \%)$, then gentamicin having a percentage of 7.5 (Figure 1).

The control group had 40 specimen samples from which only 4 organisms were isolated. All of the 4 isolates were resistant against vancomycin, clindamycin, amoxicillin, penicillin- $G$, nalidixic acid and metronidazole. Least resistance was observed against gentamicin, amikacin, and imipenem ( $0 \%$ ) (Figure 2).
Analysis of survey according to the questionnaire: The Majority of the patients were female with a percentage of 62 , while the percentage of male patients was 38 (Figure 3). From the percentage distribution of age groups of oral cancer patients, it can be seen that oral cancer is more prevalent in the age group of 40-70 (Figure 4). The geographical distribution of oral cancer patients (Figure 5) has shown that the highest number of cancer patients from this survey belonged to the Greater Mymensingh zone. The distribution of predisposing factors in oral cancer patients (Figure 6) has shown that almost $90 \%$ of patients had regular uptake of betel nut and betel leaf.

Table 1. Summary of growth of the isolates on selective media

\begin{tabular}{|c|c|c|c|c|c|c|c|}
\hline \multirow[t]{3}{*}{ Patient status } & \multicolumn{6}{|c|}{ Media } & \\
\hline & \multicolumn{3}{|c|}{$\begin{array}{c}\text { Cetrimide } \\
\text { Colony appearance }\end{array}$} & \multicolumn{4}{|c|}{$\begin{array}{c}\text { EMB } \\
\text { Colony appearance }\end{array}$} \\
\hline & $\overline{\text { Green }}$ & Lightgreen & Nogrowth & Purple/pinkmucoid & Greensheen & Colorlesslush & Nogrowth \\
\hline Post-op & 5 & 2 & 4 & 6 & - & - & 5 \\
\hline Pre-op & 18 & 2 & 8 & 30 & 4 & 8 & 8 \\
\hline Controlgroup & - & 2 & 38 & 1 & 1 & - & 38 \\
\hline
\end{tabular}

Table 2. Identification of isolates from biochemical test results

\begin{tabular}{|c|c|c|c|c|c|c|c|c|c|c|c|c|c|c|c|c|c|c|}
\hline \multirow{2}{*}{ 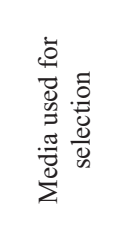 } & \multicolumn{2}{|c|}{ MR-VP } & \multirow[b]{2}{*}{$\frac{\mathscr{E}}{\frac{\pi}{\pi}}$} & \multirow[b]{2}{*}{ 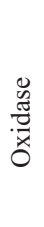 } & \multirow[b]{2}{*}{ 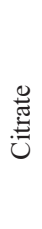 } & \multirow[b]{2}{*}{ 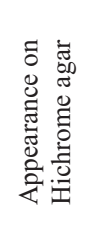 } & \multicolumn{3}{|c|}{ MIU } & \multicolumn{2}{|c|}{$\begin{array}{c}\text { Gram } \\
\text { Staining }\end{array}$} & \multicolumn{6}{|c|}{ TSI } & \multirow[b]{2}{*}{$\begin{array}{l}\text { Presumptive } \\
\text { Name of the } \\
\text { organism }\end{array}$} \\
\hline & 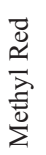 & 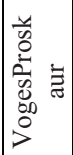 & & & & & : & $\frac{\stackrel{0}{\circ}}{\frac{0}{E}}$ & 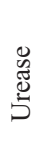 & $\frac{5}{0}$ & $\frac{\mathscr{E}}{\tilde{E}}$ & 壱 & 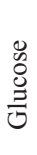 & 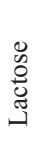 & 感 & 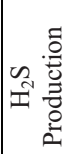 & 胥 & \\
\hline EMB & - & + & + & - & + & Blue & - & - & + & Pink & Rod & $\mathrm{Y} / \mathrm{Y}$ & + & + & + & - & + & Klebsiellaspp \\
\hline Cetrimide & - & - & + & + & + & $\begin{array}{l}\text { Light } \\
\text { Green }\end{array}$ & + & - & - & Pink & Rod & $\mathrm{R} / \mathrm{R}$ & - & - & - & - & + & Pseudomonasspp \\
\hline EMB & + & - & + & - & - & Purple & + & + & - & Pink & Rod & $\mathrm{Y} / \mathrm{Y}$ & + & + & + & - & + & Escherichia coli \\
\hline EMB & + & - & + & - & + & $\begin{array}{l}\text { Light } \\
\text { Brown }\end{array}$ & + & - & + & Pink & Rod & $\mathrm{R} / \mathrm{Y}$ & + & - & - & + & + & Proteusspp \\
\hline
\end{tabular}

Y= Yellow; R= Red; “+”= Positive; "-"= Negative

Cancer patients group

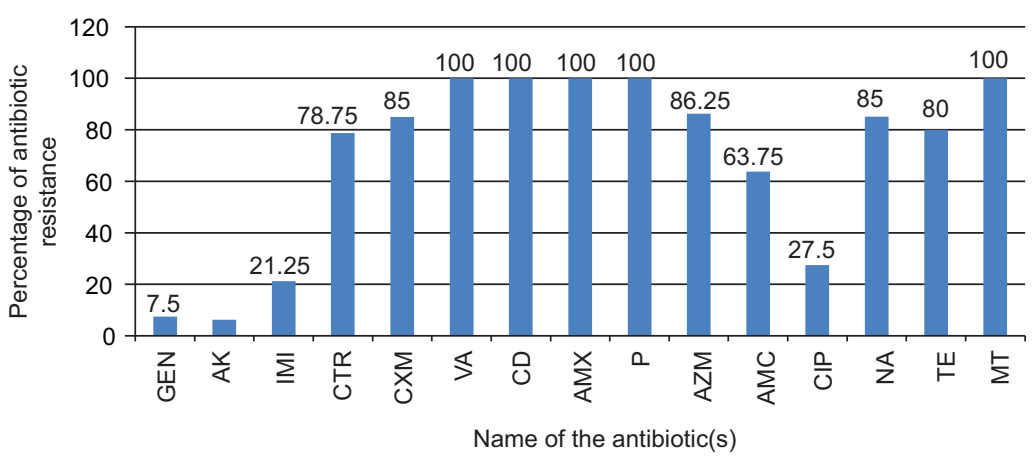

Figure 1. Antibiotic susceptibility pattern of the isolates (cancer patient group). 
Ashreen et. al.

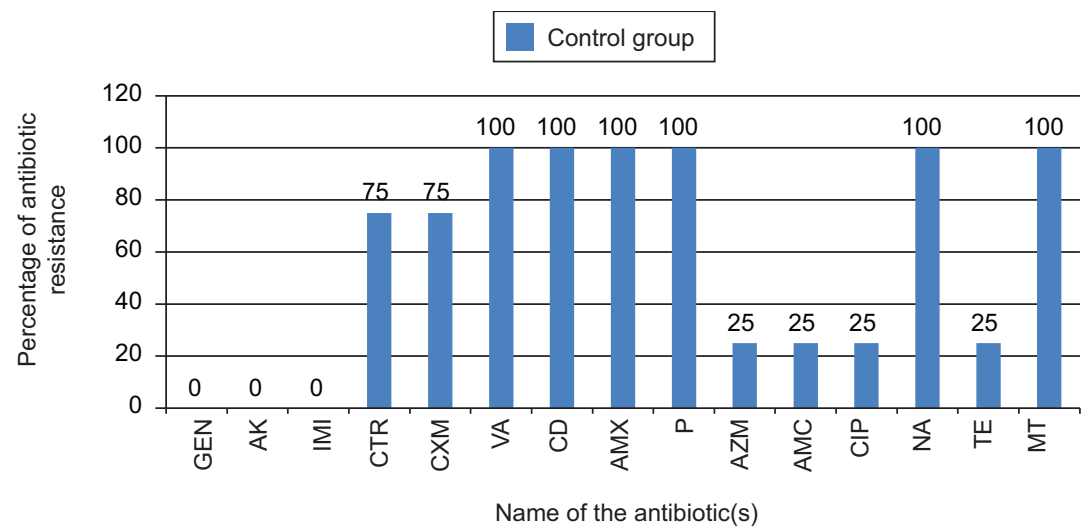

Figure 2. Antibiotic susceptibility pattern of the isolates (control group).

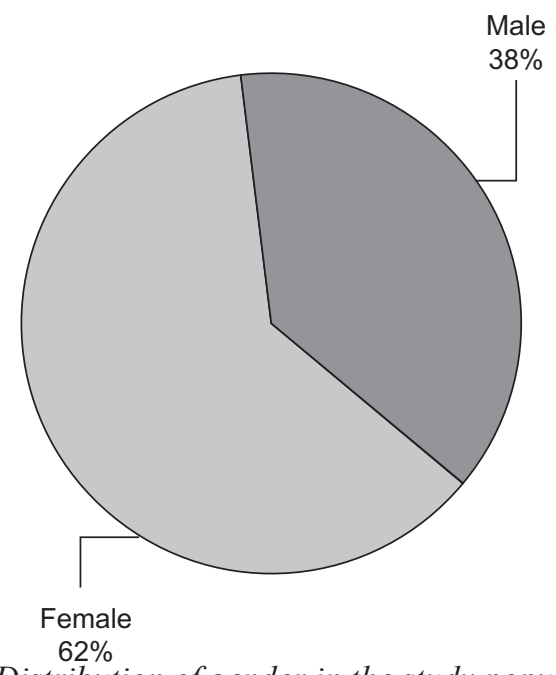

Figure 3. Distribution of gender in the study population.

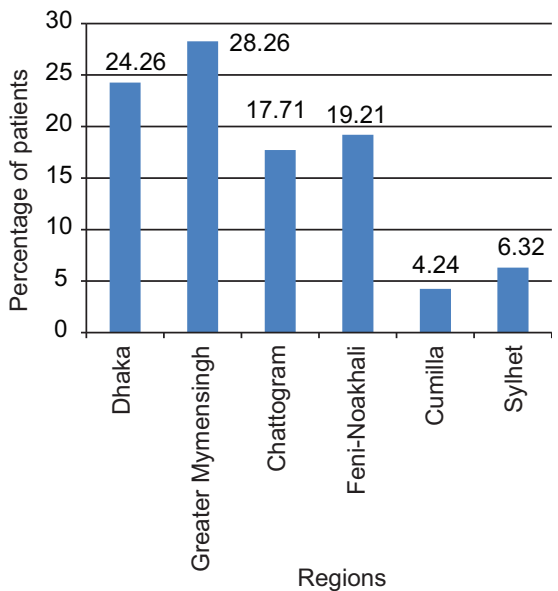

Figure 5. Geographical distribution of the study population.

\section{Discussion}

The study aimed to isolate and identify a range of opportunistic gram-negative bacteria from the ulcerative lesions of oral cancer

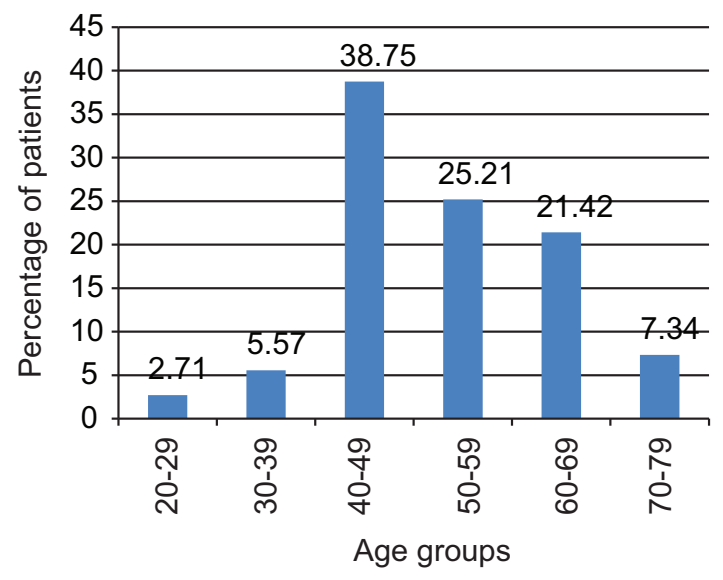

Figure 4. Distribution of age groups in the study population.

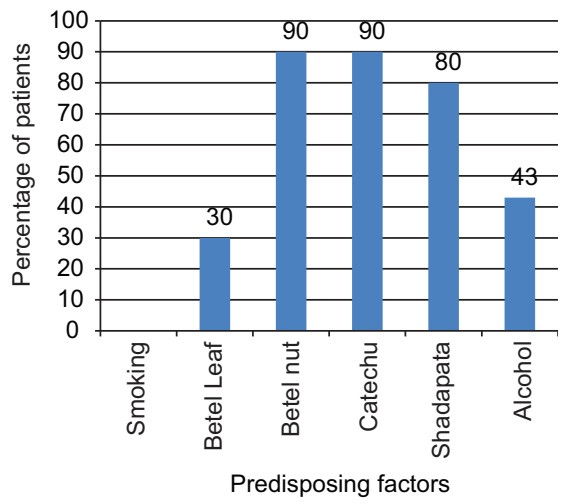

Figure 6. Distribution of predisposing factors in the study population.

patients. Opportunistic bacteria selectively colonize in the sites of ulceration in oral cavity cancer patients which result in a negative impact in the healing of those ulcer sites. The study 
represents the prevalence of gram-negative bacilli on oral cavity cancer sites and their emerging resistance patterns against different classes of antibiotics.

It is important to have knowledge of the type of pathogens that inhabit the oral cavity to prevent dental diseases as well as the associated systemic complications caused by them ${ }^{14}$. Due to the heterogeneous nature of the oral tissues and structures, it provides a diverse ecological habitat to the oral microorganisms that are distributed in various parts of the oral cavity along with the mucosal surfaces, teeth and saliva ${ }^{15}$. The organisms that were screened in this study from both the control group and test group were Pseudomonas species, Klebsiella species, Escherichia coli, and Proteus species. These opportunistic pathogens infect the host taking advantage of the weakened immune system, altered microbiota or damaged integumentary system. Drug-resistant opportunistic infections may cause health problems in immunocompromised hosts ${ }^{16}$, thus it creates various complexities in oral cancer patients.

In the performed study, among the 80 Gram-negative bacteria isolated from the 50 cancer patients, the most prevalent was Klebsiella spp. (48\%). In several studies, it was found out that 3$7 \%$ of nosocomial infections were due to this species ${ }^{17}$. Among immunosuppressed people such as cancer patients, an increasing rate of pneumonia infection has been observed ${ }^{18}$. In the samples from post-operative patients, the percentage of Klebsiella sp. isolates was higher than in pre-operative patients. Again, this shows evidence of hospital-associated infections since $K$. pneumoniae is often spread by the hands of health care workers or contaminated medical devices ${ }^{19}$. Moreover, antibiotic treatment amplifies the risk of $K$. pneumoniae infection ${ }^{20}$.

In the conducted study, out of 50 swab samples from cancer patients, Pseudomonas had a prevalence of $36 \%$. This value shows similarity with another study conducted in Western European hospitals where $P$. aeruginosa was one of the most common organisms, constituting $29 \%$ of all Gram-negative isolates ${ }^{21}$. However, in regards to isolates from pre-operative patients, the percentage of Pseudomonas was higher in this study.

The next most prevalent organism was Proteus species with a percentage of 10. Apart from the normal flora, Proteus is also found in several environmental habitats, including long-term care facilities and hospitals. A study has shown that Proteus infections generally occur as community-acquired infections, ninety percent of which is caused by P. mirabilis ${ }^{22}$.

In this study E. coli, $5 \%$ of the isolates belonged to this bacteria .Although $E$. coli is reported as one of the most major causes of nosocomial infections, in this study the $E$. coli isolates were only found in pre-operative patients.

In comparison, 40 swab samples were collected from the control group consisting of healthy volunteers, from where only 4 bacteria were isolated including 2 Pseudomonas spp., 1 Klebsiella spp. and $1 \mathrm{E}$. coli isolate. The presence of these microbes in the control group indicates probabilities of lack of practicing good oral hygiene. The bacterial isolates obtained from the control group could be sourced to the dental plaque ${ }^{23}$. Accumulation of these microbes in the oral cavity can also be attributed to some diseases or conditions that perturb the balance of the host immune system and have an effect on the host oral microbiota.

In contrast, positive results can be observed from the control group due to several factors. In a similar study, it was reported that immunocompromised patients with hematological malignancies showed that the prevalence of Enterobacteriaceae in the oral cavity was significantly higher ${ }^{24}$. This indicates possible reasons for the vast difference in the percentage of prevalent microbes isolated from oral samples of both the groups. The pro-inflammatory nature of oral cancers permits the growth of opportunistic pathogens in the oral cavity after the alteration of normal microbiota ${ }^{25}$. In patients subjected to radiotherapy, oral colonization and infection to opportunistic pathogens are observed $^{26}$.

After assessing the results from antibiotic susceptibility testing, it was shown that all the isolates from cancer patients were resistant to Penicillin group antibiotics, which included amoxicillin and penicillin G. Similar results were observed for the antibiotics from glycopeptide (vancomycin) and lincosamide (clindamycin) groups. Moreover, all the isolates showed resistance against the metronidazole antibiotic. These emerging problems of antibiotic resistance in opportunistic pathogens can cause infectious morbidity among some groups of immunecompromised patients.

Gram-negative aerobes such as Enterobacteriaceae are inherently resistant against clindamycin due to their impervious structure of the cell wall. Virtually all the anaerobic Gram-negative rods are known to be susceptible to metronidazole ${ }^{27}$. In contrast, in this study, all the microbial isolates from both cancer patients and the control group showed resistance against metronidazole antibiotics. The appearance of metronidazole resistance in microbial species that recovered from dental infections was noted in a similar study ${ }^{28}$. This emerging resistance of Gram-negative bacteria against metronidazole can be defined by the occurrence of specific resistance genes ( $\mathrm{nim}$ ) which code for an alternative set of enzymes that can convert activated forms of metronidazole into non-toxic derivatives ${ }^{29}$.

In terms of azithromycin, the second-highest resistance pattern was observed among the isolates with a percentage of 86.25. Although azithromycin has been considered to be very effective against Enterobacteriaceae infections ${ }^{30}$, mutations in the outer membrane of Escherichia coli were reported to decrease the MICs of azithromycin ${ }^{31}$.

About $21 \%$ of the total isolates from this study showed resistance against imipenem. This is lower compared to many studies where the resistance level was found up to $57 \% \%^{32}$. The quinolone showed a higher efficiency profile against the gram-negative pathogens, having a susceptibility percentage of 72.5 to ciprofloxacin. This 
is in contrast to the study carried out in India ${ }^{33}$ where $82 \%$ and 96\% of the Enterobacteriaceae isolates were resistant to ciprofloxacin and gentamicin respectively. In this study, the isolates exhibited the least resistance against amikacin (6.25\%) and gentamicin $(7.5 \%)$, both of which belong to the aminoglycoside group of antibiotics. The aminoglycosides, especially amikacin, remain the drugs active against the largest number of Klebsiella strains $^{34}$.

The rapid evolution of bacteria has been contributing to microbial drug resistance which has a relevant impact in terms of morbidity, mortality and healthcare-associated costs ${ }^{35}$.

Regarding the antibiotic resistance, combination therapy of antibiotics can be a suitable alternative to treat the opportunistic gram-negative bacteria. Moreover, the genes responsible for the resistance should be investigated via molecular techniques. Most importantly, that would allow faster detection of antibiotic resistance. Hence, it will provide a quicker administration of the most suitable drug.

\section{References}

1. Bray F, Ferlay J, Soerjomataram I, Siegel RL, Torre LA and Jemal A. 2018. Global Cancer Statistics 2018: GLOBOCAN estimates of incidence and mortality worldwide for 36 cancers in 185 countries. CA Cancer J Clin. 68(6): 394-424.

2. Parkin DM, Bray F, Ferlay J and Pisani P. 2002. Global Cancer Statistics, 2002. CA Cancer J Clin. 55(2): 74-108.

3. Ahmed, F and Islam KM. 1990. Site predilection of oral cancer and its correlation with chewing and smoking habit e A study of 103 cases. Bangladesh Med Res Coun Bull. 16(1): 17-25.

4. Shah FD, Begum R, Vajaria BN, Patel KR, Patel JB, Shukla SN and Patel PS. 2011. A review on salivary genomics and proteomics biomarkersin oral cancer. Indian J Clin Biochem . 26(4): 326-334.

5. Ram H, Sarkar J, Kumar H, Konwar R, Bhatt MLB and Mohammad S. 2011. Oral cancer: Risk factors and molecular pathogenesis. J Maxillofac Oral Surg. 10(2): 132-137.

6. Cawson RA. 1969. Leukoplakia and oral cancer. Proc Royal Soc Med. 62(6): 610-615.

7. Shaheed SMI and Molla MR. 1996. Oral cancer in Bangladesh: Its aetiology and histological grading. $J$ Oral Health. 2: 8-11.

8. Sultana N and Malik M. 2014. The overview of oral cancer and risk factors in Bangladesh. Int J Dental Sci Res. 2(5A): 8-10.

9. Kumar M, Nanavati R, Modi TG and Dobariya C. 2016. Oral cancer: Etiology and risk factors: A review. J Can Res Ther. 12: 458-463.

10. Senpuku H, Sogame A, Inoshita E, Tsuha Y, Miyazaki H and Hanada N. 2003. Systemic disease in association with microbial species in oral biofilm from elderly requiring care. Gerontology. 49: 301-309.

11. Cappuccino JG and Sherman N. 1996. The Microbiology - A Laboratory Manual, $4^{\text {th }}$ edn. The Benjamin/Cummings Publishing Co., Inc., Menlo Park, California.

12. CLSI. 2009. Performance standards for antimicrobial susceptibility testing; $19^{\text {th }}$ informational supplement. CLSI M100-S19 Clinical and Laboratory Standards Institute, Wayne, Pennsylvania.

13. Ferraro MJ, Craig WA and Dudley MN. 2001. Performance standards for antimicrobial susceptibility testing. USA:NCCLS, Pennsylvania.

14. Philip K, Teoh WY, Muniandy S and Yaakob H. 2009. Pathogenic bacteria predominate in the oral cavity of Malaysian subjects. J Biol Sci. 9: 438444.

15. Gaonkar PP, Patankar SR, Tripathi N and Sridharan G. 2018. Oral bacterial flora and oral cancer: The possible link? J Oral Maxillofac Pathol. 22(2): 234-238.
16. Yamashita K, Ohara M, Kojima T, Nishimura R, Ogawa T, Hino T, Okada M, Toratani S, Kamata N, Sugai M and Sugiyama M. 2013. Prevalence of drug-resistant opportunistic microorganisms in oral cavity after treatment for oral cancer. J Oral Sci. 55(2): 145-155.

17. Horan TC, Garner JS, Jarvis WR, Emori TG and Hughes JM. 1988. CDC definitions for nosocomial infections. Am J Infect Contr. 16(3): 128-140.

18. Carpenter CB. 1990. Immunosuppression in organ transplantation. N Engl J Med. 322(17): 1224-1226.

19. Gupta A. 2002. Hospital-acquired infections in the neonatal intensive care unit d Klebsiella pneumoniae. Semin Perinatol. 26: 340-345.

20. Nguyen ML, Toye B, Kanji S and Zvonar R. 2015. Risk factors for and outcomes of bacteremia caused by extended-spectrum beta-lactamaseproducing Escherichia coli and Klebsiella species at a Canadian tertiary care hospital. Can J Hosp Pharm. 68: 136-143.

21. Vincent JL, Rello J, Marshall J, Silva E, Anzueto A, Martin CD, Moreno R, Lipman J, Gomersall C, Sakr Y and Reinhart K. 2009. International study of the prevalence and outcomes of infection in intensive care units. JAMA. 302: 2323-2329.

22. Wang S, Zhang Y, Zhang X and Li J. 2019. An evaluation of multidrugresistant (MDR) bacteria in patients with urinary stone disease: Data from a high-volume stone management center. World J Urol. DOI: 10.1007/ s00345-019-02772-0.

23. Rocio RC, Florence LG, Krista R, Gilles R, Michèle V, Stephanie G, Geneviève H, Georges B and Sylvie B. 2015. Pseudomonas aeruginosa and periodontal pathogens in the oral cavity and lungs of cystic fibrosis patients: A case control study. J Clin Microbiol. 53(6): 1898-1907.

24. Bergmann OJ. 1988. Oral infections and septicemia in immunocompromised patients with hematologic malignancies. J Clin Microbiol. 26: 2105-2109.

25. Idris A, Hasnain SZ, Huat LZ and Kohac D. 2017. Human diseases, immunity and the oral microbiota e Insights gained from metagenomic studies. Oral Sci Int. 14(2): 27-32.

26. Leung WK, Jin LJ, Yam WC and Samaranayake LP. 2001. Oral colonization of aerobic and facultatively anaerobic Gram-negative rods and cocci in irradiated, dentate, xerostomic individuals. Oral Microbiol Immunol. 16(1): 1-9.

27. Dhand A and Snydman DR. 2009. Mechanism of resistance in metronidazole. In Antimicrobial Drug Resistance. Infectious Disease (Mayers DL ed). Humana Press, New York.

28. Smith A. 2018. Metronidazole resistance: A hidden epidemic? BDJ. 224: 403-404.

29. Leiros HK, Kozielski-Stuhrmann S, Kapp U, Terradot L, Leonard GA and McSweeney SM. 2004. Structural basis of 5-nitroimidazole antibiotic resistance: The crystal structure of NimA from Deinococcus radiodurans. J Biol Chem. 279: 55840-55849.

30. Gomes C, Roldán LR, Mateu J, Ochoa TJ and Ruiz J. 2019. Azithromycin resistance levels and mechanisms in Escherichia coli. Scientific Reports 9, Article number: 6089.

31. Vaara M. 1993.Outer membrane permeability barrier to azithromycin, clarithromycin, and roxithromycin in Gram-negative enteric bacteria. Antimicrob Agents Chemother. 37(2): 354-356.

32. Teo JQ, Cai Y, Lim TP, Tan TT and Kwa AL. 2016. Carbapenem resistance in Gram-negative bacteria: The not-so-little problem in the little red dot. Microorganisms. 4: E13.

33. Khan MKR, Thukral SS and Gaind R. 2008. Evaluation of a modified double disc synergy test for detection of extended spectrum ${ }^{2}$-lactamases in AmpC ${ }^{2}$-lactamase producing Proteus mirabilis. Indian $J$ Med Microbiol. 26: 58-61.

34. Smith SM, Digori JT and Eng RH. 1982. Epidemiology of Klebsiella antibiotic resistance and serotypes. J Clin Microbiol. 16(5): 868-873.

35. Cosgrove SE. 2006. The relationship between antimicrobial resistance and patient outcomes: Mortality, length of hospital stay, and health care costs. Clin Infect Dis. 42(Suppl. 2): 82-89. 